\title{
HOMEM IDOSO QUE TRABALHA NO CAMPO: VULNERABILIDADES INDIVIDUAIS, SOCIAIS E PROGRAMÁTICAS
}

\author{
Lucimare Ferraz ${ }^{1}$ \\ Ligiane Pauly ${ }^{2}$ \\ Rosana Maria Badalotti ${ }^{3}$ \\ Marta Kolhs ${ }^{4}$
}

Resumo: O processo de envelhecimento e masculinização no meio rural é um fenômeno mundial. Esse fato gera diversas situações que necessitam ser (re)conhecidas pela sociedade pública e privada. Esse artigo tem por objetivo tencionar uma reflexão sobre o homem idoso trabalhador rural por suas vulnerabilidades, em três dimensões: individual, a social e a pragmática. Tratase de uma revisão narrativa que buscou na literatura contemporânea descrever e analisar o corpo de conhecimento sobre a temática. Por fim, evidenciou-se que os homens idosos rurais estão em situação de vulnerabilidade, uma vez que encontram-se isolados socialmente; além de trabalharem em ambientes insalubres e perigosos devido suas deficiências físicas e psíquicas; outrossim, há limitações em exercerem seus direitos enquanto trabalhadores, aposentados e cidadãos.

Palavras-chave: Homem. Idoso. Trabalhador. Rural. Vulnerabilidade.

\begin{abstract}
The process of aging and masculinization in rural areas is a worldwide phenomenon. This fact generates several situations that need to be (re) known by public and private society. The objective of this article is to reflect on the rural working elderly man
\end{abstract} for his vulnerabilities, in three

\footnotetext{
${ }^{1}$ Doutora em Ciências da saúde (Unifesp). Docente do Programa de Pós-graduação em Ciências da Saúde e em Enfermagem.

${ }^{2}$ Enfermeira. Universidade do Estado de Santa Catarina- UDESC.

${ }^{3}$ Doutora em Interdisciplinar em Ciências Humanas (2003) pela Universidade Federal de Santa Catarina. Professora titular do Programa de Pós-Graduação em Políticas Sociais e Dinâmicas RegionaisUnochapeco.

${ }^{4}$ Doutora Enfermagem UFRGS Docente da Universidade do Estado de Santa Catarina (UDESC) na graduação e pós-graduação "lato sensu"
} 
dimensions: individual, social and pragmatic. It is a narrative review that sought in contemporary literature to describe and analyze the body of knowledge on the subject. Finally, it was shown that rural elderly men are vulnerable, since they are socially isolated; besides working in unhealthy and dangerous environments due to their physical and mental deficiencies; there are also limitations in exercising their rights as workers, retirees and citizens.

Keywords: Man. Old man. Worker. Rural. Vulnerability.

\section{Contextualizando}

No Brasil, e em outros países, o meio rural passa por transformações produzidas, essencialmente, pelo êxodo rural seletivo com predomínio de jovens e mulheres, caracterizando processos de masculinização e envelhecimento populacional no campo (ANJOS, CALDAS, 2005).

Esse fenômeno de masculinização do campo, já foi descrito na década de 60 na França, na obra 'El Baile de los Solteiros' de Bourdieu (2004). Segundo Bourdieu (2004, apud COSTA, FROEHLICH; CARPES,
2013) o processo de masculinização rural implica na diminuição da presença feminina neste contexto, comprometendo a composição familiar, e consequentemente a sucessão dos estabelecimentos.

Pesquisas recentes sobre a demografia rural têm apontado a existência de relação indireta entre a modernização da agricultura e a intensidade da masculinização rural, a partir da diminuição drástica do grau de intensidade de utilização da terra e da mão de obra, que têm ocorrido em algumas atividades agropecuárias modernizadas (COSTA, FROEHLICH; CARPES, 2013)

Neste sentido, se torna fundamental analisar tal fenômeno recorrente no Brasil há mais de vinte anos, como consequência da modernização agrícola que têm gerado impactos na migração de jovens, sobretudo mulheres, desestabilizando a composição geracional equilíbrio social nas áreas rurais, gerando o processo de masculinização do campo (COSTA, MATOS, VALLE, 2015). Este processo social que desequilibra a composição populacional rural em termos de idade e gênero e cuja recorrência vem sendo 
relatada não só no Brasil, mas também em vários outros países latinoamericanos e europeus, é a recorrência do patriarcado ser mais evidente nas condições rurais, atribuindo culturalmente ao homem o papel produtivo, do trabalho na lavoura e na lida campeira (COSTA, FROEHLICH, 2014).

Além da tendência de masculinização do campo, pelo êxodo rural feminino, tem se observado um aumento da idade mediana dos agricultores, ocasionando o envelhecimento da população rural (ANJOS, CALDAS E POLLNOW, 2014). Idoso permanece no campo se responsabilizando, muitas vezes, pelas atividades agropecuárias da propriedade.

Os homens idosos trabalhadores rurais desempenham as mais diversas atividades: desde o preparo do solo para plantio, colheita, transporte e armazenagem de produtos e insumos agrícolas, controle de pragas nas plantações, aplicação de agrotóxicos e fertilizantes, além de inúmeras atividades específicas desenvolvidas paralelamente, como abertura de canais de irrigação, drenagem e manutenção (MARTINS; FERREIRA, 2015).
A Organização Internacional do Trabalho (OIT) afirma que a atividade laboral na agricultura é significativamente mais perigosa que outras atividades e estima que milhões de agricultores sofram sérios problemas de saúde (OIT, 2006). A agricultura apresenta um ambiente insalubre para os trabalhadores e essa condição é agravada quando se trata do idoso, devido às implicações e limitações orgânicas da idade (FERRAZ; ALVES; FERRETI, 2017).

Nesse contexto, as vulnerabilidades do homem idoso trabalhador rural vão além dos aspectos fisiopatológicos da idade e do processo laboral, apontando para uma realidade em que predomina a pobreza, isolamento, baixos níveis educacionais, residências mais precárias, limitações de transporte, problemas crônicos de saúde e distância dos recursos sociais e de saúde, dentre outras (MORAIS; RODRIGUES; GERHARDT, 2008).

Ayres e colaboradores (2012) descrevem $\quad 0$ termo vulnerabilidade como, à chance de exposição das pessoas ao adoecimento, sendo resultante de um conjunto de aspectos relacionados ao indivíduo, a 
comunidade e a nação. O conceito de vulnerabilidade trouxe uma concepção ampliada e reflexiva sobre promoção da saúde e bem-estar, passando por aspectos comportamentais, culturais, econômicos e políticos (AYRES et al., 2003).

A vulnerabilidade pode ser dividida em três dimensões interdependentes que abordam elementos pessoais/vulnerabilidade individual, da comunidade/ vulnerabilidade social e de políticas/vulnerabilidade programática. A vulnerabilidade individual refere-se à qualidade da informação que cada indivíduo dispõe sobre possíveis danos e a capacidade de transformar essas informações em práticas de prevenção. Quanto à vulnerabilidade social, aborda as questões de acesso a informações e aos serviços de saúde, recursos sociais, políticas, bens culturais, direito a democracia, condições de bem-estar entre outros. A vulnerabilidade programática trata das questões políticas e institucionais que, através do comprometimento e monitoramento das ações, visam prevenir agravos na da população (AYRES et al., 2012).

O declínio biológico, as limitações de interações socioculturais, as condições deficitárias de educação, renda e saúde; assim como o estilo de vida e a relação com o trabalho (RODRIGUES; NERI, 2011), faz dos homens idosos trabalhadores rurais uma população vulnerável. Essa vulnerabilidade não ocorre somente pelo processo de envelhecimento somando tarefas pouco estruturadas, a exposição de produtos químicos, condições ambientais desfavoráveis e os fatores ergonômicos como postura inadequada e grande esforço físico (POLETTO FILHO, 2013).

Neste trabalho destaca-se o homem idoso trabalhador rural por suas vulnerabilidades, segundo os preceitos de Ayres et al., (2012), que descreve as situações de vulnerabilidade em três dimensões: individual, a social e a pragmática. Metodologicamente se trata de uma revisão narrativa, que segundo Mattos (2015) é uma abordagem de pesquisa que não utiliza critérios explícitos e sistemáticos para a busca e análise crítica da literatura. $\mathrm{O}$ autor cita que esta forma de investigação não objetiva esgotar as fontes de informações e também não se utiliza de uma única estratégia de busca. Desta forma, os conteúdos e interpretação das 
informações estão sujeitas à delimitação subjetividade dos pesquisadores.

Para Rother (2007) a revisão narrativa se constitui de conteúdos da literatura em livros, revistas impressas e/ou eletrônicas e baseia-se na interpretação e análise crítica pessoal do autor. Esse estudo teve como fontes de pesquisa livros, artigos, teses e dissertações que abordam temas como: Gerontologia, Saúde coletiva, Saúde ocupacional, Vulnerabilidades em Saúde; Saúde do Trabalhador; Saúde do Idoso; Masculinização no Campo.

\section{Vulnerabilidade Individual}

Vulnerabilidade individual está relacionada ao modo de vida das pessoas; trata-se da autonomia e conhecimento para interpretar informações de saúde e doença e as transforma em práticas efetivas de prevenção (AYRES et al., 2012).

O processo de envelhecimento é complexo e pode ser descrito em fases distintas, a cronológica, fisiológica e funcional. A idade cronológica baseiase quantidade de anos que a pessoa viveu já a idade fisiológica diz respeito às funções corporais, porém não é possível prever quando estas ocorrerão e também a idade funcional que se refere à capacidade de contribuir para a sociedade ou a si próprio, sendo que nem todos os indivíduos da mesma idade cronológica tem o mesmo nível funcional (BRUM, 2005). O envelhecimento se caracteriza pela diminuição progressiva da função orgânica do organismo e diminuindo a manutenção do equilíbrio homeostático, sendo um processo individual, natural e fisiológico (MAIA, 2005).

No contexto da vulnerabilidade individual, o homem idoso trabalhador rural está exposto a inúmeros agravos devido à perda funcional comum da idade, somados aos riscos ocupacionais presentes no ambiente rural, aqui apresentados segundo a Norma Regulamentadora (NR) 9, a saber: riscos físicos, biológicos, ergonômicos, acidentes e químicos. Os riscos variam de acordo com sua natureza, intensidade, suscetibilidade e tempo de exposição. A seguir detalharemos cada um dos elementos/aspectos da vulnerabilidade individual no idoso trabalhador rural.

Os agentes físicos são formas de energias que expõem o trabalhador, aqueles que interferem diretamente 
sobre o mesmo (MARTINS; FERREIRA, 2015). Em relação aos agentes físicos, destaca-se a exposição excessiva a radiação solar, sendo uma rotina comum nas atividades agrícolas o trabalho sob o sol (COUTO, 2016). O idoso trabalhador rural é mais vulnerável a exposição à radiação solar devido ao processo natural e fisiológico do envelhecimento, que acarreta na diminuição progressiva da função orgânica do organismo, diminuindo a manutenção do equilíbrio homeostático (MAIA, 2005). Destaca-se o câncer de pele uma vez que a exposição à radiação solar é ainda mais prejudicial em idosos devido às alterações cronológicas da pele, decorrente de uma diminuição de melanócitos funcionais que sintetizam a melanina responsável pela proteção da pele contra raios ultravioletas (LUECKENOTTE, 2002).

Assim como o câncer de pele, a exposição ao sol pode levar o idoso ao processo de desidratação. Esta moléstia decorre de uma série de fatores como: acentuado declínio da quantidade de água corpórea, redução dos componentes intracelulares e extracelulares, diminuição da sede acarretando na redução da ingesta hídrica, pela termorregulação comprometida em variações extremas de temperatura, decréscimo do número de glândulas sudoríparas e pela perda da capacidade de reter a umidade (DUARTE; DIOGO, 2005).

As mudanças bruscas de temperatura de um ambiente quente para um ambiente frio provocam alterações, sendo prejudiciais à saúde. Ambientes quentes podem ocasionar no aumento da temperatura superficial da pele, sudação, tonturas e desmaios, taquicardia, hipotensão ou mal-estar. Já os ambientes frios são igualmente prejudiciais, e levam à alteração circulatória do sangue, queimaduras de frio e doenças do aparelho respiratório (SOUZA, 2005).

Em relação às doenças do aparelho respiratório, chama-se a atenção quanto a poeiras e umidades que podem ser agentes nocivos, pois o idoso apresenta um progressivo declínio funcional e estrutural tanto no tórax como nos pulmões, a parede dos alvéolos torna-se mais fina e fibrosa, há uma redução da complacência da parede torácica pela rigidez e calcificação das cartilagens costais, as bases dos pulmões não inflam bem e as secreções não são facilmente expectoradas propiciando 
riscos a infecções. Essas mudanças na capacidade pulmonar deixam o idoso mais suscetível a disfunções respiratórias (LUECKENOTTE, 2002).

Outro fator para vulnerabilidade do homem idoso trabalhador rural é a introdução de maquinários na agricultura. Esses maquinários podem causar efeitos de vibrações resultando em complicações musculoesqueléticas, as quais o idoso já sofre alterações relacionadas a idade, afetando ossos, músculos e articulações. Com a idade o corpo sofre perda progressiva da massa óssea, enfraquecendo os ossos; as fibras dos músculos esqueléticos de degeneram, levando a redução de massa, tônus e força muscular; encolhimento de tendões; calcificação da cartilagem articular, afetando a amplitude de movimento e de marcha, essas alterações resultam em diminuição da capacidade funcional musculoesquelética (LUECKENOTTE, 2002).

O ruído presente nos maquinários agrícolas, também pode ocasionar uma situação de vulnerabilidade ao idoso, pois pode ocasionar distúrbios ao nível de sistema nervoso, desconfortos, irritabilidade, cefaleia ou até mesmo levando a surdez que pode ser temporária, surdez definitiva e trauma acústico (SOUZA, 2005).

Os agentes biológicos são aqueles capazes de causar danos à saúde do trabalhador, como infecções, efeitos tóxicos, efeitos alergênicos, doenças autoimunes e a formação de neoplasias e malformações. Esses agentes são microrganismos geneticamente modificados, toxinas, parasitas, animais e plantas que podem causar efeitos alergênicos, irritativos ou tóxicos (BRASIL, 2008). Esses microrganismos estão na terra, em adubos orgânicos e na água, promovendo a ocorrência de enfermidades infecciosas e verminoses. Ainda no meio rural é importante considerar os agentes biológicos com alta probabilidade de transmissão de doenças como dengue, brucelose, leptospirose ou doenças virais (MARTINS; FERREIRA, 2015; ARAÚJO; GOSLING; HARDOIM, 2007).

O risco biológico à saúde do homem idoso trabalhador rural, se dá pelo fato de estar mais vulnerável às alterações funcionais e morfológicas nas células que compõem o sistema imune. O processo de envelhecimento do sistema imune chama-se de 
imunossenescência. Trata-se de um processo complexo, continuo e de início não estabelecido, que acarreta em diferentes fatores clínicos como o aumento da susceptibilidade a infecções, retarda à recuperação de doenças, reativação de infecções latentes, menor proteção de vacinas, maior tendência ao desenvolvimento de certos tumores e doenças autoimunes (KINOSHITA, 2014).

\section{Os agentes ergonômicos são} aqueles relacionados a fatores fisiológicos e psicológicos inerentes à atividade laboral. Esses agentes podem produzir alterações no organismo e no estado emocional dos trabalhadores, comprometendo sua saúde, segurança e produtividade (PEIXOTO, 2010).

As atividades agrícolas exigem dos trabalhadores idosos deslocamentos constantes em extensas áreas, agachamentos, rotações e flexões de tronco, longos períodos em pé, esforço físico intenso e postura em movimento, com os membros superiores constantemente elevados acima do nível dos ombros, além de carregamento de peso (MARTINS; FERREIRA, 2015).

As atividades laborais no campo requerem dos trabalhadores esforços físicos excessivos, comprometendo as condições de saúde do idoso, que pelo processo de envelhecimento sofre alterações musculoesqueléticas deixando-os mais suscetíveis à riscos ergonômicos (ALVES; FERRETI; FERRAZ, 2017).

Em relação às alterações esqueléticas destaca-se a osteoporose, caracterizada pela perda progressiva da massa óssea e deterioração do tecido esquelético. $\mathrm{O}$ efeito da diminuição do tecido ósseo é o enfraquecimento, os ossos ficam porosos aumentando o risco a fratura na terceira idade (LUECKENOTTE, 2002; ROACH, 2003).

Quanto às mudanças articulares, com a idade ocorre uma diminuição do liquido sinovial que tem a função de lubrificar as articulações, a cartilagem torna-se mais fina e os ligamentos podem ficar curtos e menos flexíveis. Essas alterações resultam na diminuição da amplitude e movimento (LUECKENOTTE， 2002; ROACH, 2003), bem como geram dores articulares. Já as alterações musculares se dão pela diminuição das fibras, a perda do tônus muscular os tornando mais finos e flácidos, resultando em 
fraqueza e rigidez que contribui para a limitação dos movimentos (LUECKENOTTE， 2002; ROACH, 2003).

Os agentes de acidentes são aqueles decorrentes das condições físicas e tecnológicas, impróprias, capazes de provocar lesões à integridade física do trabalhador. Estes riscos são decorrentes do uso de ferramentas e de máquinas em condições precárias de segurança (SANTOS, 2007).

Os homens idosos são mais vulneráveis aos acidentes pela debilidade musculoesquelética relacionada à idade, uma das principais alterações do processo de envelhecimento. Tal deficiência afetam a capacidade funcional e contribuem para o risco de quedas em virtude das mudanças na postura e do centro de gravidade, diminuição do tempo e reação e da força muscular do idoso (LUECKENOTTE, 2002). Um dos principais riscos de acidentes que 0 trabalhador idoso está exposto são as quedas, isso devido a modificações atribuídas ao processo natural de envelhecimento e a diversas patologias, podendo afetar a capacidade funcional deste trabalhador. A queda, geralmente, é responsável pelas perdas da autonomia e da independência do idoso (SILVA et al., 2007).

Os riscos de queda presentes na agricultura vão desde as condições do solo e das estradas que geralmente não são pavimentadas e apresentam riscos significativos pelas pedras ou cascalhos soltos propiciando quedas ou escorregões, ou ainda pelos animais na pista que podem causar acidentes de traumas. Outros fatores como maquinários ou ferramentas, cercas e alguns objetos que sejam de difícil percepção ao idoso, podendo propiciar um ambiente hostil á saúde (MARCHIORI; FERRAZ, 2014).

Outro fator para a vulnerabilidade aos acidentes com idosos é a baixa acuidade visual e auditiva, essas mudanças sofridas com a idade levam a alterações múltiplas estruturais desses órgãos resultando na perda progressiva sensorial (INÁCIO, 2011). Acuidade visual além de um fator para quedas, assim como a acuidade auditiva apresenta outros riscos de acidentes pela diminuição de informações sensoriais e o ajuste postural inadequado (DUARTE; DIOGO, 2005). Essas mudanças de 
percepção e lentidão de informações propiciam riscos nas atividades laborais no meio rural (MARTINS; FERREIRA, 2015).

Os agentes químicos são aqueles representados pelas substâncias líquida, sólida e gasosa e em contato com o organismo podem produzir reações tóxicas e danos à saúde (SANTOS, 2007). Quanto ao uso de agentes químicos na agricultura, destaca-se o uso de agrotóxico. Destacando que o Brasil é considerado campeão mundial no uso de agrotóxicos, com uma média de mais de cinco quilos para cada brasileiro por ano, segundo o Dossiê da Associação Brasileira de Saúde Coletiva (ABRASCO, 2015). Os danos causados pelos agrotóxicos à saúde variam com a exposição, podendo ocorrer pelas vias, oral, inalatória e ou dérmica, acarretando problemas ao homem idoso, como intoxicações, cânceres, doenças de pele, gástricas e respiratórias (ABRASCO, 2015).

O idoso está mais vulnerável as condições de intoxicações por agrotóxicos devido à falta de informação e treinamento técnico, pelo baixo nível de escolaridade, por dificuldades no manuseio dos produtos, dificuldades de
10

acesso à saúde e amparo social e o não uso de equipamentos de proteção individual

(EPI's) adequadamente/satisfatoriamente

(ALVES; FERRETI; FERRAZ, 2017).

A vulnerabilidade individual do homem idoso trabalhador rural pode ser minimizada com algumas práticas e medidas, como o uso de Equipamentos de Proteção Individual - EPI's, e os de proteção coletiva - EPC's, assim como, capacitações para o autocuidado.

\section{Vulnerabilidade Social}

A vulnerabilidade social analisa os aspectos que expõem os indivíduos ao risco social. Nessa perspectiva, aborda questões que envolvem a vulnerabilidade individual como, isolamento social, acesso à saúde, a escolarização, conhecimento e informações, a renda, disponibilidade de recursos materiais, enfim aspectos que garantam a integração social (AYRES et al., 2012).

A vulnerabilidade social entre a população idosa está relacionada a diversidade de cenários enfrentados no cotidiano, aos aspectos culturais, sociais, econômicos, de saúde, entre outros 
(BARBOSA, 2015). Na situação de vulnerabilidade social do idoso trabalhador rural, ressaltam-se as questões de insalubridades de seu trabalho na agricultura. Muitos idosos, devido sua situação financeira, permanecem na atividade laboral agrícola ou retornam a ela após a aposentadoria, pela necessidade de uma renda adicional (MAGALHÃES, 2008; VANZELLA et al., 2011).

No Brasil, o baixo valor das aposentadorias está entre os fatores da vulnerabilidade social, pois expõe não apenas a pessoa idosa, mas todo o grupo familiar que sobrevive com esse recurso. Devido às altas taxas de desemprego e subempregos, o idoso passa a contribuir com sua aposentadoria para o sustento da família, tornando-se muitas vezes, o principal provedor da família. Porém nem sempre as aposentadorias conseguem promover condição social adequada, ao alcance de uma boa qualidade de vida na terceira idade (AEROSA, 2008; PAZ; SANTOS; EIDT, 2006).

No que se refere a questão de vulnerabilidade social-econômica, chama-se a atenção para o consumo de medicamentos que encarece o padrão de vida, pois o idoso é mais suscetível a múltiplas doenças, sendo comum nessa fase da vida o consumo de grandes quantidades de medicamentos, levando a um declínio de sua condição socioeconômica (DUARTE; DIOGO, 2005). Diante desse fato, o agricultor idoso acaba permanecendo na agricultura e se mantém ativo na produção da lavoura para contribuir nas necessidades do lar através da produção para o autoconsumo como hortas, criação de pequenos animais e pequenas plantações (FERRAZ, ALVES; FERRETI; 2017).

Outra dificuldade voltada aos idosos que residem em áreas rurais é a questão do acesso aos serviços de saúde que devido a distância, dificuldade de locomoção, precariedade das estradas e ao isolamento geográfico estão ainda mais vulneráveis a agravos de saúde (MORAIS;

RODRIGUES;

GERHARDT, 2008).

A procura por serviços de saúde é ocasional ou apenas em casos mais graves, pois o trabalhador rural idoso geralmente não tem quem o substitua em suas tarefas cotidianas, e sua ausência implica no acúmulo de 
O distanciamento dos serviços de saúde é amenizado pelo trabalho das Equipes da Saúde da Família (ESF), que é fundamental na assistência à saúde dessa população. Essa assistência é prestada através de visitas domiciliares com o objetivo de realizar atividades de educação e promoção de saúde, estabelecer vínculos de compromisso e corresponsabilidade, estimular a organização das comunidades, entretanto, essas atividades também são afetadas pelas dificuldades de acesso dos agentes de saúde as residências rurais (DIMPÉRIO et al., 2009).

Essa condição de vulnerabilidade do homem idoso rural é agravada pela questão do isolamento social. O próprio processo de envelhecimento contribui para aumentar esta vulnerabilidade, pois se trata de uma fase de conflitos, devido à diminuição do status profissional, das relações sociais e afetivas, o que vai exigir um esforço do idoso em se adaptar a essas situações (TEIXEIRA, 2010).

No que se refere ao isolamento social do homem idoso rural, se destaca a carência de momentos para interação social. Programações sociais, como grupos de terceira idade, para a população idosa no meio urbano é comum e de fácil acesso. Os grupos de convivência de idosos possibilitam à interação social e geram benefícios a saúde e social (WICHMANN, 2009). Contudo este tipo de organização é mais frequente no meio urbano.

\section{Vulnerabilidade Programática}

Vulnerabilidade programática refere-se as políticas sociais, que estão imbricadas, e que geram impactos, nos aspectos individuais e sociais dos indivíduos e comunidades (AYRES et al., 2012). No campo da vulnerabilidade programática, se destacam políticas para a população idosa em termos mais gerais e específicas no que se refere à saúde desta população.

A Política Nacional do Idoso, instituída pela lei $\mathrm{N}^{\circ} 8.842$ em janeiro de 1994, tem como objetivo assegurar os direitos sociais do idoso, criando condições para promover sua autonomia, integração e participação efetiva na sociedade, sendo considerada idosa a pessoa com sessenta anos ou mais (BRASIL, 1994). Entretanto, as ações de promoção da saúde e de prevenção de doenças, e mesmo de atendimento domiciliário, ainda necessitam ser 
ISSN | 2179-7137 | http://periodicos.ufpb.br/ojs2/index.php/ged/index

ampliadas (ou implementadas) para atender aos princípios da Política Nacional de Saúde da Pessoa Idosa, proporcionando independência e autonomia por mais tempo possível (BARBOSA, 2015).

Outro documento que abrange e assegura a população mais envelhecida é o Estatuto do Idoso, firmado pela Lei $\mathrm{N}^{\circ}$ 10.741 de outubro de 2003 e é destinado a regular os direitos assegurados pela pessoa idosa. Nessa Lei o idoso trabalhador tem seus direitos trabalhistas assegurados no Cap. VI, a saber:

Art. 26. O idoso tem direito ao exercício de atividade profissional, respeitadas suas condições físicas, intelectuais e psíquicas.

Art. 27. Na admissão do idoso em qualquer trabalho ou emprego, é vedada a discriminação e a fixação de limite máximo de idade, inclusive para concursos, ressalvados os casos em que a natureza do cargo o exigir: Parágrafo único. $\mathrm{O}$ primeiro critério de desempate em concurso público será a idade, dando-se preferência ao de idade mais elevada.

Art. 28. O poder público criará e estimulará programas de:

I - profissionalização especializada para os idosos, aproveitando seus potenciais e habilidades para atividades regulares e remuneradas; II preparação dos trabalhadores para a aposentadoria, com antecedência mínima de um ano, por meio de estímulo a novos projetos sociais, conforme seus interesses, e de esclarecimento sobre os direitos sociais e de cidadania; III - estímulo às empresas privadas para admissão de idosos ao trabalho (BRASIL, 2003).

No campo da Saúde Coletiva, em outubro de 2006, portaria $\mathrm{N}^{\mathrm{o}} 2.528$, o Ministério da Saúde do Brasil a prova a Política Nacional de Saúde da Pessoa Idosa, com o objetivo de recuperar, manter e promover a autonomia e a independência dos indivíduos idosos, direcionando medidas coletivas e individuais de saúde para esse fim, em consonância com os princípios e diretrizes do Sistema Único de Saúde (BRASIL, 2006).

Em dezembro de 2011, pela portaria $\mathrm{N}^{\mathrm{o}} 2.866$, o $\mathrm{MS}$ instituiu a Política Nacional de Saúde Integral das Populações do Campo e da Floresta (PNSIPCF). Esta política tem o objetivo de promover a saúde das populações do campo e da floresta, visando ao acesso aos serviços de saúde, à redução de riscos e agravos à saúde decorrente dos processos de trabalho e das tecnologias agrícolas e à melhoria dos indicadores de saúde e da qualidade de vida (BRASIL, 2011). 
No ano de 2012, o MS

implementa a Política Nacional de Saúde do Trabalhador, instituída na Lei, $\mathrm{n}^{\circ}$ 1.823 de 23 de agosto de 2012, tendo como objetivo definir os princípios, as diretrizes e as estratégias a serem observados nas três esferas de gestão do SUS - federal, estadual e municipal, para o desenvolvimento de ações que garantam aos trabalhadores, atenção integral à saúde (BRASIL, 2012). Contudo, essa Politica não aborda questões específicas voltadas ao homem idoso trabalhador e ao trabalhador idoso rural.

Observa-se a necessidade de ações programáticas para essa população, que está vulnerável pelas dificuldades inerentes de viver e trabalhar no meio rural, pela ausência de articulações multissetoriais e esforços programáticos através de políticas específicas (AYRES et al., 2012).

\section{Considerações Finais}

Por fim, observa-se que o homem idoso trabalhador rural está vulnerável individualmente devido ao processo orgânico de envelhecimento, resultando no declínio da capacidade funcional
(PAZ; SANTOS; EIDT, 2006), assim como os fatores de riscos relacionados ao processo laboral agrícola. Segundo Martins e Ferreira (2015), o trabalhador rural está exposto aos mais diversos riscos ocupacionais. Além disso, baixo nível de escolaridade e a falta de conhecimento quanto ao uso de EPI's e o manuseio de maquinários, torna essa população vulnerável aos acidentes e as consequências sociais dessas injurias (FERRAZ, ALVES;FERRETTI; 2017).

Quanto à vulnerabilidade social, percebem-se dificuldades quanto as baixas aposentadorias, o uso de medicações levando ao declínio da condição econômica, a dificuldade de acesso aos serviços de saúde e de comunicação entre os setores sociais. Sobre esse aspecto Ferreira (2015), destaca o despreparo dos profissionais que atuam nesse meio, o desconhecimento sobre o território e suas demandas, a pouca permanência dos profissionais nesses locais, dificultando uma relação de vínculo com essa população.

Em relação a vulnerabilidade programática, percebe-se que o homem idoso trabalhador rural, está desassistido de programas, ações e políticas 
específicas voltadas a essa população (ALVES; FERRETI; FERRAZ, 2014). É imprescindível que os profissionais da Saúde Coletiva e dos Serviços de Assistência Social, que atuam no meio rural, considerem a imbricação dos elementos ambientais, sociais e culturais nos determinantes e condicionantes do processo saúde-doença dessa população (FERREIRA, 2015).

$\mathrm{Na}$ agricultura a vulnerabilidade do homem idoso trabalhador rural vai além do processo de envelhecimento, ressalta-se a questão da modernização agrícola, à grande utilização de ferramentas e implementos, maquinário e ao uso, cada vez maior de agrotóxicos, favorecendo um ambiente hostil ao trabalhador (MARTINS; FERREIRA, 2015).

Outra questão presente no meio rural são as condições de pobreza, isolamento, baixos níveis educacionais, residências precárias, limitações de transporte, problemas crônicos de saúde e distância dos recursos sociais e de saúde, dentre outras (MORAIS; RODRIGUES; GERHARDT, 2008). Nesse sentido, vale destacar que o índice alto de analfabetismo no meio rural, a falta de transporte; 0 estado problemático das estradas, precariedade das infraestruturas, as dificuldades de acesso, o isolamento social e o desamparo de políticas (FURTADO, 2016), a masculinização da população do campo (BOURDIEU, 2004; ANJOS, CALDAS, 2005, COSTA, FROEHLICH; CARPES, 2013; ANJOS, CALDAS E POLLNOW, 2014; COSTA, MATOS, VALLE, 2015), propiciam condições de vulnerabilidades ao homem idoso trabalhador rural.

\section{Referencias Bibliograficas}

AREOSA, Silvia Virginia Coutinho. Novas configurações familiares a partir do idoso como provedor. Pontifícia Universidade Católica do Rio Grande do Sul Porto Alegre. 2008, pp. 212.

ABRASCO. Um alerta sobre os impactos dos agrotóxicos na saúde. Rio de Janeiro. 2015.

ANJOS, Flávio Sacco Dos; CALDAS, Nádia Velleda; POLLONOW, Germano Ehlert. "Menos mulheres, menos jovens, mais incertezas: a transição demográfica no Brasil rural meridional". Rev. Extensão Rural. 2014, pp. 94-116. 
ANJOS, Flávio Sacco dos; CALDAS, Nádia Velleda. "O futuro ameaçado: o mundo rural face os desafios da masculinização, envelhecimento e desagrarização". Ensaios FEE, Porto Alegre, 2005, pp.661-694.

ARAÚJO, Gilberto Cifuentes Dias; GOSLING, Marlusa; HARDOIM, Paulo César. Segurança do trabalhador rural. Agroanalysis. 2007, pp 30-40.

AYRES, José Ricardo de Carvalho Mesquita et al. O Conceito de Vulnerabilidade e as Práticas de Saúde: novas perspectivas e desafios. In: CZERESNIA, Dina; FREITAS, Carlos Machado de. Tratado de Saúde Coletiva. São Paulo: Hucitec, 2012, pp. 176.

AYRES, José Ricardo de Carvalho Mesquita et al. Risco, vulnerabilidade e práticas de prevenção e promoção da saúde. In: CAMPOS, Gastao Wagner de Sousa; CARVALHO,Yara Maria de; MINAYO,Maria Cecilia de Souza; DRUMOND Junior,Marcos ; AKERMAN, Marco. Tratado de Saúde Coletiva. São Paulo: Hucitec, 2012, pp. 976.
BARBOSA, Keylla Talitha Fernandes. Vulnerabilidade física, social e programática de idosos atendidos na atenção primária de saúde do município de João Pessoa. Paraíba. 2015.

BRASIL. Lei $\mathrm{n}^{\circ} 10.741$, de $1^{\circ}$ de outubro de 2003.: Dispõe sobre o Estatuto do Idoso e dá outras providências.

BRASIL. PORTARIA N ${ }^{\circ} 1.823$, DE 23

DE AGOSTO DE 2012. 2012.

BRASIL. Ana Maria Resende Chagas. Saúde e segurança no trabalho no Brasil: Aspectos Institucionais, Sistema de Informações e Indicadores. Ipea (Org.). 2011.

BRASIL. Portaria n. ${ }^{\circ} 2.528$ de 19 de outubro de 2006. 2006.

BOURDIEU, Pierre. El baile de los solteros. Barcelona: Anagrama, 2004, pp. 282.

COSTA, Cassiane da; FROEHLICH, José Marcos; CARPES, Ricardo Howes. "Masculinização rural: uma abordagem a partir da regionalização por sistemas agrários no Rio Grande do Sul”. Rev. 
bras. estud. popul. São Paulo, 2013, pp.

465-483.

COSTA, Alfredo; MATOS, Ralfo Edmundo da Silva; VALLE, Matheus Henrique Fernandes. "Análise dos processos de masculinização no meio rural dos municípios brasileiros segundo porte populacional e grau de modernização de agropecuária". Rev. de geo. agra. 2015, pp. 271-292.

COSTA, da Cassiane; FROEHLICH, Marcos José. "Políticas públicas e masculinização rural no Rio Grande do Sul - uma abordagem a partir das condições regionais". Rev. de geo. agra. 2014, pp. 27-54.

COUTO, José Luiz Viana. Riscos de acidentes na zona rural. Rio de Janeiro, 2006.

DIMPÉRIO, Maida Geni Siqueira et al. Saúde rural:o caso da linha das flores. Sober. Porto Alegre. 2009, pp. 00 - 12.

DUARTE, Yeda Aparecida de Oliveira; DIOGO, Maria José D'Elboux. Atendimento domiciliar: um enfoque gerontológico. São Paulo. Atheneu, 2005, pp. 630.
FERRAZ, Lucimare. ALVES, Jessica; FERRETTI, Fatima. “A vulnerabilidade ocupacional do idoso no meio rural". Sau. \& Transf. Soc. Florianópolis. 2017.

INÁCIO, Candice Cristina Fiúza. Prevenção de Queda em Idosos: possibilidades da garantia de um envelhecimento saudável. Salvador. 2011, pp. 55.

KINOSHITA, Denise. "Alterações do sistema imunológico relacionadas ao envelhecimento e suas consequências". Rev. da Universidade Ibirapuera. São Paulo. 2014.

LUECKENOTTE, Annette. Avaliação em Gerontologia. São Paulo. 2002, pp. 286.

MARTINS, Anameire de Jesus; FERREIRA, Nilza Sampaio. "A ergonomia no trabalho rural". Rev. Eletrôn. Atualiza Saúde, Salvador. 2015.

MAIA, Flávia de Oliveira Motta. Fatores de Riscos para Óbitos em Idosos. Rev. esc. enferm. São Paulo, 2005. 
MARCHIORI, Paula Marco; FERRAZ,

Lucimare. Análise da vulnerabilidade gestacional de trabalhadoras rurais assistidas pela Estratégia Saúde da Família. Chapecó, 2014, pp.173.

MORAIS, Eliane Pinheiro de; RODRIGUES, Rosalina Aparecida Partezani; GERHARDT, Tatiana Engel. "Os idosos mais velhos no meio rural: realidade de vida e saúde de uma população do interior gaúcho”. Texto Contexto Enferm. Florianópolis. 2008, pp. 374-83.

PAZ, Adriana Aparecida; SANTOS, Beatriz Regina Lara dos; EIDT, Olga Rosaria. "Vulnerabilidade e envelhecimento no contexto da saúde". Rev. Acta Paul Enferm. Vacaria. 2006, pp.338-342.

PEIXOTO, Neverton Hofstadler.

Segurança do Trabalho I. Santa Maria. 2010, pp. 152.

POLETTO FILHO, José Antonio. "Análise dos Riscos Físicos e Ergonômicos em Roçadora Transversal Motorizada". Revista da Associação Brasileira de Ergonomia. Botucatu, 2013, pp. 70-81.
RODRIGUES, Natália Oliveira; NERI, Anita Liberalesso. "Vulnerabilidade social, individual e programática em idosos da comunidade: dados do estudo FIBRA". Ciênc. saúde coletiva. São Paulo. 2011.

ROTHER, Edna Terezinha. Revisão sistemática $\mathrm{X}$ revisão narrativa. Acta paul. enferm. São Paulo, 2007.

SOUSA, Jerônimo et al. Acidentes de Trabalho e Doenças Profissionais em Portugal Riscos Profissionais: Fatores e Desafios. 2005, pp. 70.

SILVA, Tatiana Magalhães et al. "A vulnerabilidade do idoso para as quedas: análise dos incidentes críticos". Rev. Elet. de Enferm. Goiás. 2007.

TEIXEIRA, Liliana Márcia Fernandes. "Solidão, depressão e qualidade de vida em idosos:um estudo avaliativo exploratório e implementação piloto de um projeto de intervenção". Lisboa. 2010.

VANZELLA, Elidio, et al. "A Terceira Idade e o Mercado de Trabalho". Revista 
Periódico do Núcleo de Estudos e Pesquisas sobre Gênero e Direito Centro de Ciências Jurídicas - Universidade Federal da Paraíba Edição Especial - Health, Gender and Human Rights V. 7 - No 01 - Ano 2018

ISSN | 2179-7137 | http://periodicos.ufpb.br/ojs2/index.php/ged/index

Brasileira de Ciências da Saúde. João

Pessoa. 2011.

WICHMANN, Francisca Maria

Assmann et al. "Grupos de convivência

como suporte ao idoso na melhoria da saúde”. Rev. Bras. Geriatr. Gerontol. Rio

de Janeiro. 2009, pp. 821-832. 